\title{
Knockdown of MTDH increases drug sensitivity to HDAC inhibitor and TRAIL combination treatment in endometrial cancer cells
}

\author{
Xiangbing Meng, ${ }^{1,2}$ Pavla Brachova, ${ }^{1}$ Shujie Yang, ${ }^{1}$ Zhi Xiong,,${ }^{1}$ Yuping Zhang, ${ }^{1}$ \\ Kim Leslie ${ }^{1,2}$
}

Key words: MTDH, Metadherin, TRAIL, LBH 589, HDAC

Understanding the molecular mechanisms of chemoresistance is vital to design therapies to restore chemosensitivity. In particular, metadherin (MTDH) has been demonstrated to have a critical role in chemoresistance. Over-expression of MTDH has recently been correlated with poor clinical outcomes in breast cancer, neuroblastoma, hepatocellular carcinoma and prostate cancer. In endometrial cancer patients, lymph node metastasis and increase of mortality were significantly associated with chromosome 8q22-q23copy number gains, which is where MTDH is localized. However, it is unclear how MTDH overexpression mechanistically contributes to endometrial cancer development, progression, or response to therapy. In this present study, we detected overexpression of MTDH in endometrial cancer tissues compared to normal endometrium. Studies in endometrial cancer cell lines indicate that MTDH depletion enhanced sensitivity to chemotherapy agents as well as cell death induced by a combinatorial treatment of histone deacetylase (HDAC) inhibitor and tumor necrosis factor-alpha-related apoptosisinducing ligand (TRAIL) . TRAIL promotes death of cancerous cells of the human reproductive tract, and HDAC inhibitors, such as LBH 589 used in this study, have been shown to increase sensitivity of cancer cells to TRAIL-induced apoptosis. Inhibition of PI3K/AKT survival pathway such as PDK1 and AKT phosphorylation, increased degradation of anti-apoptosis protein XIAP, and increased expression of pro-apoptosis protein $\mathrm{Bim}$ and cleaved active isoforms of caspase 8 and caspase 3 were all observed during cell death induced by TRAIL and LBH 589 combination treatment in MTDH depleted endometrial cancer cells. However, in cancer cells with MTDH overexpression, TRAIL/LBH 589 treatment had no effect on these prosurvival factors. Interestingly, MTDH was also found to be involved in $G 2 / M$ checkpoint regulation. Treatment of MTDH-depleted cells with TRAIL and LBH589 resulted in an increased number of cells arrested in G2/M compared to cells with MTDH

${ }^{1}$ Departments of Obstetrics and Gynecology, ${ }^{2}$ Holden Comprehensive Cancer Center, The University of lowa, lowa City, lowa 52242

Corresponding author: Xiangbing Meng, Department of Obstetrics and Gynecology, University of lowa, 3234 MERF, 200 Hawkins Drive, lowa City, IA, 52242. Telephone(319) 335-8212 xiangbing-meng@uiowa.edu

This is an Open Access article distributed under the terms of the Creative Commons Attribution 3.0 Unported License (http://creativecommons.org/licenses/by/3.0), which permits unrestricted use, distribution, and reproduction in any medium, provided the original work is properly cited. 
AACR 102nd Annual Meeting, April 2 - 6, 2011,

Orlando, Florida

overexpression. Using microarray technology, 57 genes with greater than two-fold gene expression alteration were identified after MTDH depletion, including Calbindin 1 and Galectin 1, which may contribute to MTDHmediated drug resistance. Therefore, MTDH mediates drug resistance by regulating expression of genes required

for the control of apoptosis and cell cycle. These findings indicate that sensitivity to chemotherapy agents and combination treatment with HDAC inhibitor and TRAIL can be restored by manipulating $\mathrm{MTDH}$, and hence depletion of MTDH is a potentially novel avenue for effective cancer therapy.

Combination therapy in endometrial cancer 